\title{
Heat Load Measurements on a Large Superconducting Magnet: An Application of a Void Fraction Meter
}

\author{
R. Pengo, N. Dolgetta, S. Junker, G. Passardi, and H. ten Kate
}

\begin{abstract}
ATLAS is one of the two major experiments of the LHC project at CERN using cryogenics. The superconducting magnet system of ATLAS is composed of the Barrel Toroid (BT), two End Caps Toroids and the Central Solenoid. The BT is formed of 8 race-track superconducting dipoles, each one $25 \mathrm{~m}$ long and $5 \mathrm{~m}$ wide. A reduced scale prototype (named $\mathrm{BO}$ ) of one of the 8 dipoles, about one third of the length, has been constructed and tested in a dedicated cryogenic facility at CERN. To simulate the final thermal and hydraulic operating conditions, the BO was cooled by a forced flow of $4.5 \mathrm{~K}$ saturated liquid helium provided by a centrifugal pump of $80 \mathrm{~g} / \mathrm{s}$ nominal capacity. Both static and dynamic heat loads, generated by the induced currents on the B0 casing during a slow dump or a ramp up, have been measured to verify the expected thermal budget of the entire BT. The instrument used for the heat load measurements was a Void Fraction Meter (VFM) installed on the magnet return line. The instrument constructed at CERN was calibrated in order to provide direct readings of heat loads. An example of application of the VFM measuring method to a large-scale apparatus cooled at liquid helium temperature.
\end{abstract}

Index Terms-Cryogenic instrumentation, cryogenics, eddy currents, superconducting magnets.

\section{INTRODUCTION}

$\mathbf{T}$ HE ATLAS Barrel Toroid (BT), with overall dimensions of $20 \mathrm{~m}$ in diameter and $26 \mathrm{~m}$ in length, consists of eight coils, configured to produce a toroidal magnetic field for the ATLAS muon spectrometer. The coils are super-conducting, their conductor being based on a NbTi Rutherford cable, extruded in a pure $\mathrm{Al}$ cladding. The conductor is wound to form race-track shaped "double pancakes," each $25 \mathrm{~m}$ long and $5 \mathrm{~m}$ wide. One BT coil is composed of two "double pancakes" inserted in an $\mathrm{Al}$ alloy casing, and installed in an individual cryostat. The coils are indirectly cooled by a flow of liquid $\mathrm{He}$ (LHe) into pipes being glued on the coil casing. The required large flow is provided by centrifugal pumps. The thermal shields are also indirectly cooled by gas $\mathrm{He}(\mathrm{GHe})$ at about $60 \mathrm{~K}$ average temperature.

A race-track prototype model coil (about 40 tonnes), named B0, of full width but one third length $(8 \mathrm{~m})$ has been constructed

Manuscript received October 20, 2003.

R. Pengo is with CERN, Accelerator Technology division, 1211 Geneva 23, Switzerland on leave of absence from INFN-LNL, Italy (e-mail: Ruggero.Pengo@cern.ch).

N. Dolgetta is with Euratom-CEA Cadarache, F-13108 Saint-Paul-lez-Durance, France (e-mail: dolgetta@pegase.cad.cea.fr).

S. Junker and G. Passardi are with CERN, Accelerator Technology division, 1211 Geneva 23, Switzerland (e-mail: Steffen.Junker@cern.ch; Giorgio.Passardi@cern.ch).

H. ten Kate is with CERN, EP Division (e-mail: Herman.tenKate@cern.ch). Digital Object Identifier 10.1109/TASC.2004.831046

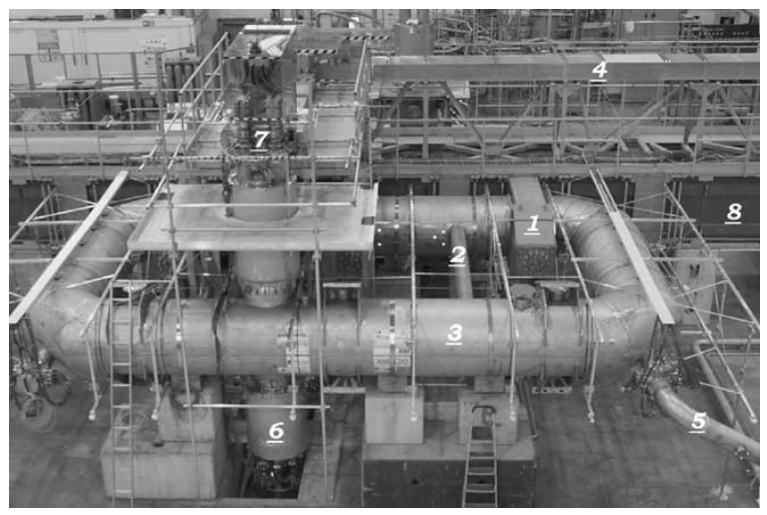

Fig. 1. Layout of B0 coil test-bench: (1) support units, (2) ribs, (3) vacuum vessel, (4) busbars system, (5) vacuum line, (6) cryogenic service turret, (7) current lead turret, (8) magnetic mirror.

and then extensively tested at CERN to validate the design criteria. The static heat loads in different conditions as well as the dynamic heat loads have been measured, using a Void Fraction Meter (VFM) developed at CERN [1]. The VFM was installed on the return line to the pump cryostat, allowing, with its fast time response, measurement of the amount of vapor produced by the electric currents induced during a fast current change. In fact eddy currents are produced in the coil casing when the magnet is charged or discharged toward dissipative elements like resistors and/or diodes.

\section{ATLAS B0 CoIl Features and Test Station}

The B0 coil [2] is shown in Fig. 1. It is placed in the test bench, in front of a "magnetic mirror", formed of iron blocks, which produces external forces of the same order as for the assembled BT coils in their final toroidal configuration. LHe at $4.5 \mathrm{~K}$ and GHe at about $40 \mathrm{~K}$ are supplied by means of flexible cryogenic transfer lines through the bottom turret, where also the electrical joints between the two double-pancakes of the coil are located. The top turret contains the current leads, which supply the current to the coil up to $24 \mathrm{kA}$.

Fig. 2 shows a cross-section of B0 and its main features. Casing and double-pancakes form the so called 'cold mass which is supported on the cryostat by sliding feet, made out of reinforced fiber glass. The electromagnetic centrifugal forces are counter-reacted by titanium tie-rods, anchored to the cryostat. LHe cooling pipes, located at "optimal" positions on the casing, maintain the cold mass at the nominal operating temperature of $4.5-4.7 \mathrm{~K}$.

Two sets of cooling pipes are installed, one set being redundant to be used in case of He leaks. 


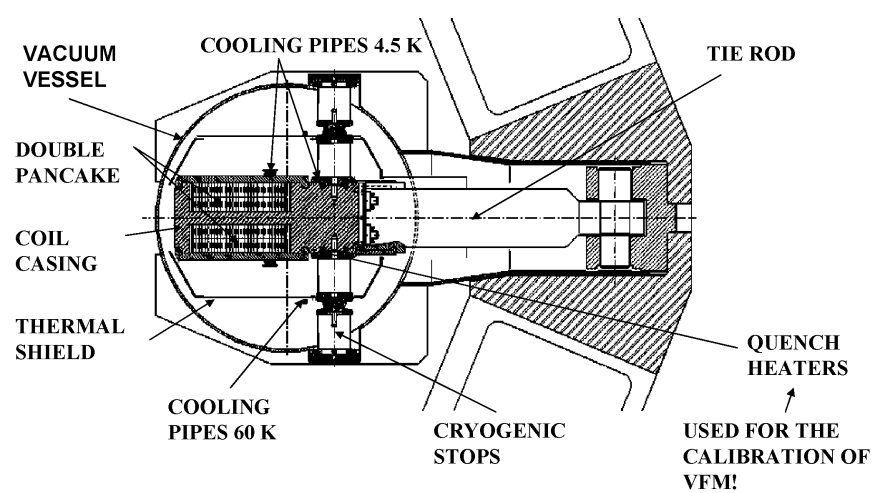

Fig. 2. The B0 cross section and the main coil features. The cross section is the same as for the final BT coil.

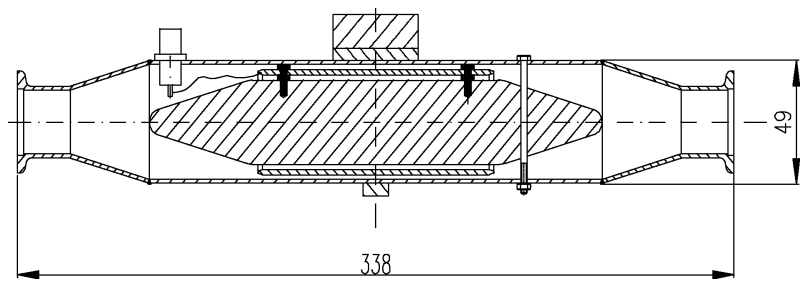

Fig. 3. The cross section of the Void Fraction Meter. Dimensions are in $\mathrm{mm}$ On the top center part is shown the location of the cold oscillator [1].

Four quench propagator resistors per coil ("quench heaters"), $0.5 \mathrm{~m}$ long, are symmetrically placed between the double pancakes (inner side) and the casing. They will be automatically switched on in case a transition in the superconductor is detected by the magnet safety system. They have been used, during the B0 heat load measurements, for the calibration of the VFM.

The test station used for the $\mathrm{B} 0$ has been described in details in [3], [4] and it will be also used to test the eight BT coils before their installation in the ATLAS cavern. The test station includes a He refrigerator ( $1200 \mathrm{~W}$ of equivalent capacity at $4.5 \mathrm{~K}$ ), which is connected to a pump cryostat. A distribution valve box allows the cryogenic connections to two BT coils. Two LHe centrifugal pumps, which are immersed in the liquid helium bath inside the cryostat, are used: one generates the forced flow through the coil cooling circuit, the second one being redundant. Before entering the coil circuits the helium flow is passed through a heat exchanger also immersed in the same bath. The return flow is sent back to the pump cryostat, which acts as a phase separator, while the refrigerator maintains the pump cryostat $\mathrm{LHe}$ level constant. During the tests the pumps delivered 80-85 g/s, at a rotational speed of $3950 \mathrm{rpm}$. The VFM was located inside the pump cryostat on the return line from the magnet.

\section{DESCRIPTION OF THE VFM AND ITS CALIBRATION}

A cross section of VFM is shown in Fig. 3. It consists of a coaxial capacitor inserted in an oscillating resonant circuit, whose resonance frequency is around $24 \mathrm{MHz}$. The working principle has been extensively described elsewhere [1]. We recall here that, due to the fact that the dielectric constant $\varepsilon$ differs of about $4 \%$ from the pure vapor to the pure liquid at $4.5 \mathrm{~K}$, the resonant frequency of the circuit changes accordingly. The dielectric constant is higher for LHe than for GHe. A higher dielectric constant $(\mathrm{LHe})$ increases the capacitance and, therefore, reduces the resonant frequency of the circuit. The value of the

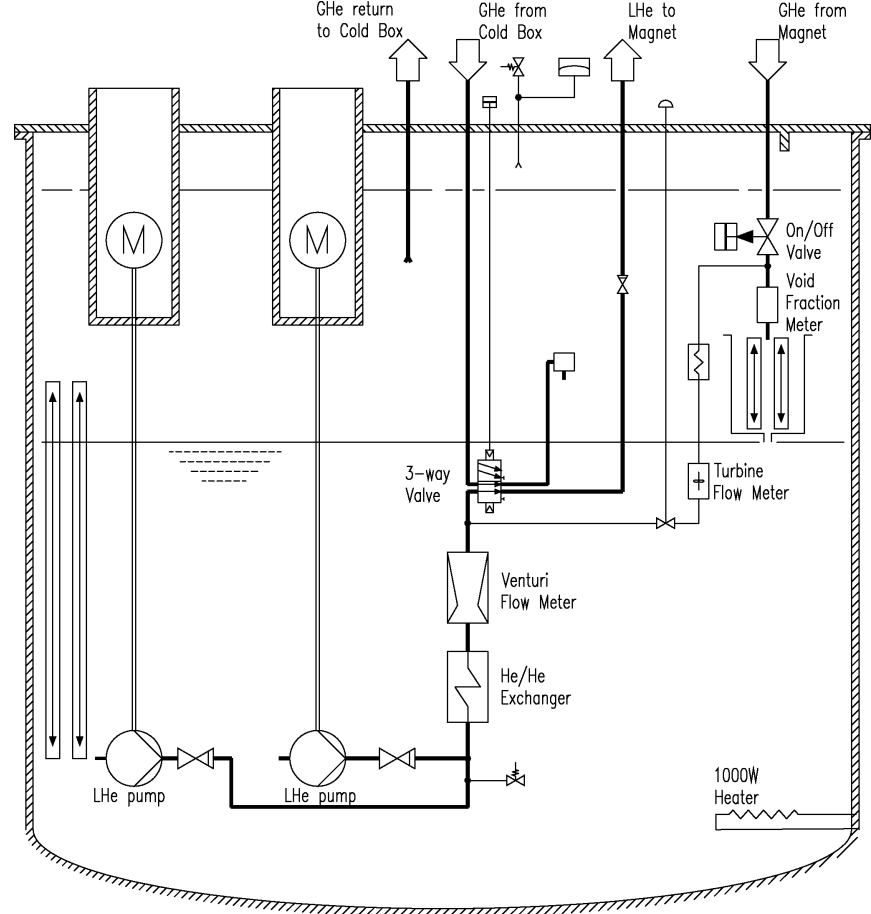

Fig. 4. The diagram of the pump cryostat with the VFM.

Calibration Curve of the VFM

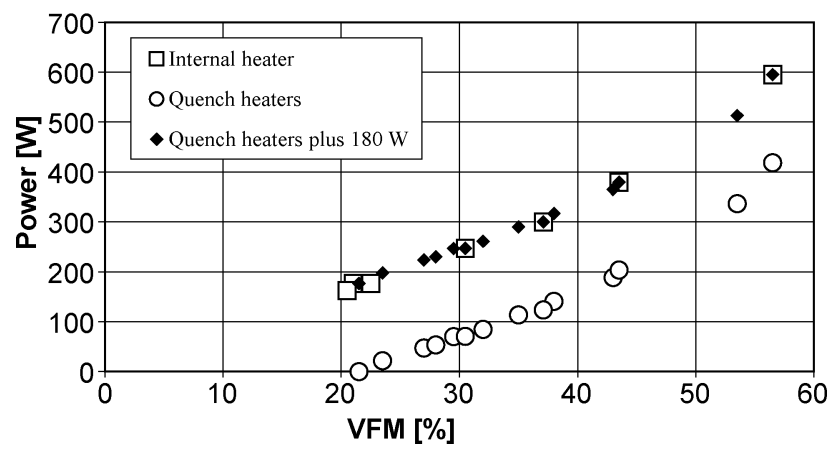

Fig. 5. The VFM calibration curve, with the thermal shield at $60 \mathrm{~K}$, as obtained by two different methods. The upper values (squares) are obtained with the internal heater. The bottom ones (circles) are obtained injecting electric power on the cold mass by means of the quench heaters. The high accuracy of the calibration is evident, since the bottom values, when increased each by $180 \mathrm{~W}$ (diamonds), just overlap on the upper values (squares).

frequency is converted into a percentage, in terms of area of the vapor compared to the total area. Due to the complexity of the two phase He system, it is not straightforward to transform the measured ratio $\alpha$ (ratio between cross sections) to the "vapor quality' $x$ (ratio between the mass flow rate of the vapor and the total mass flow rate),

$$
\alpha=\frac{A_{\text {vapor }}}{A_{\text {liquid }}+A_{\text {vapor }}} ; \quad \chi=\frac{\dot{m}_{\text {vapor }}}{\dot{m}_{\text {liquid }}+\dot{m}_{\text {vapor }}}
$$

in order to evaluate the heat load. To calibrate the instrument, we used both a heater placed in the pipe, by-passing inside the cryostat the overall facility, and the set of quench heaters on the B0 cold mass. The two calibration curves differ by a constant factor due to the heat load of the magnet and of the overall external cryogenic facility, thus validating our calibration method as shown in Fig. 5. 


\section{Signal Analysis of the VFM}
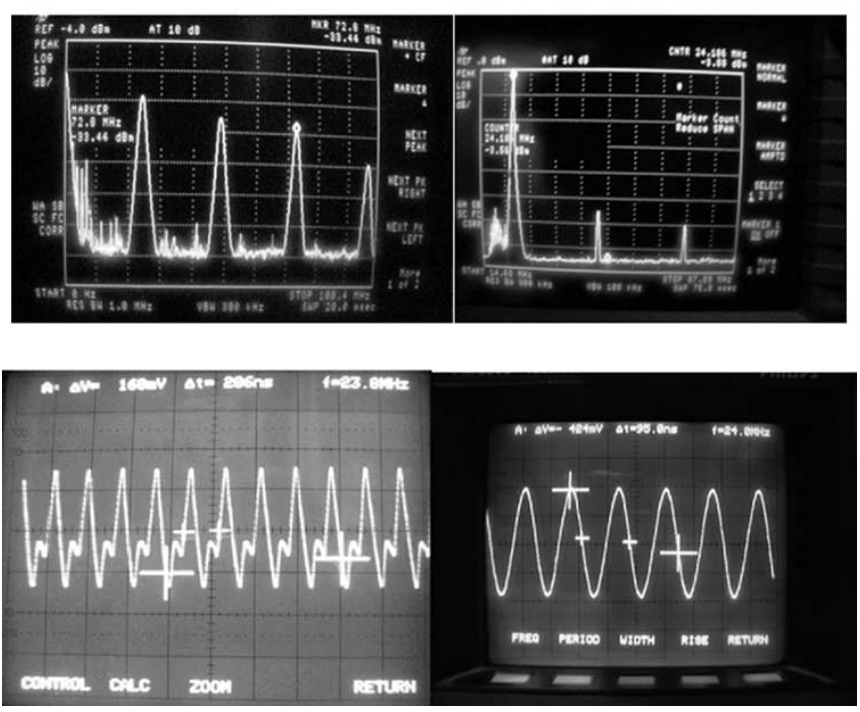

Fig. 6. Above is the spectrum analysis of the resonant circuit before (left) and after the insertion of the RF filters. A reduction of about $5 \mathrm{~dB}$ is visible. Below is the wave form before (left) and after the insertion of the RF filters (see text for details)

\section{Heat Load as a Function of the Shield Temperature}

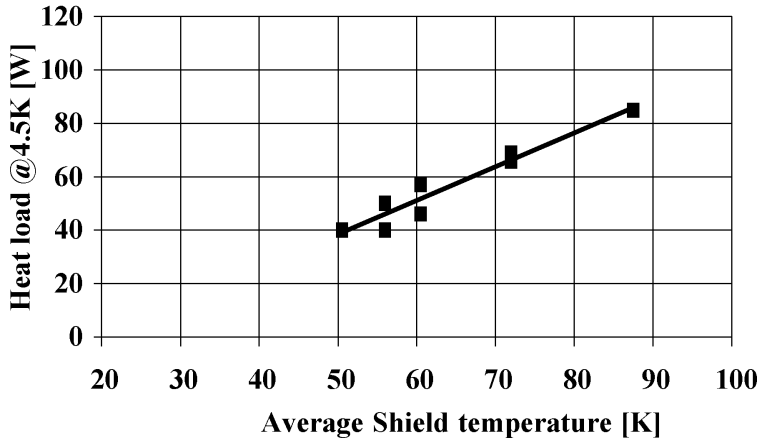

Fig. 7. Experimental values of the heat load for various shield temperatures.

During the calibration we noticed the frequent appearance of drifts in the oscillating resonant frequency as read by the digital frequency-meter. The spectrum analysis of the resonating frequency revealed the strong presence of higher-order harmonics, which resulted in a distorted sinus wave form (see Fig. 6).

The insertion of two RF filters (a notch and a low-pass type) reduced the second harmonics by $5 \mathrm{~dB}$ and brought back to normal the sinus wave form. In Fig. 6 the spectrum analysis and the corresponding resonant wave forms are shown.

\section{STATIC HeAT LOAD IN B0}

The static heat load of the magnet was measured by subtracting from the total load the value obtained by short circuiting the cryogenic lines before the entrance of the magnet. A set of data was collected changing the average temperature of the thermal shield from $50 \mathrm{~K}$ to $88 \mathrm{~K}$. The results are shown in Fig. 7. In particular it should be noticed that, at the average thermal shield temperature of $60 \mathrm{~K}$, the load is $50 \mathrm{~W}$, which is in good agreement with the calculations [5]. The almost linear trend of the values suggests that the major heat input might come

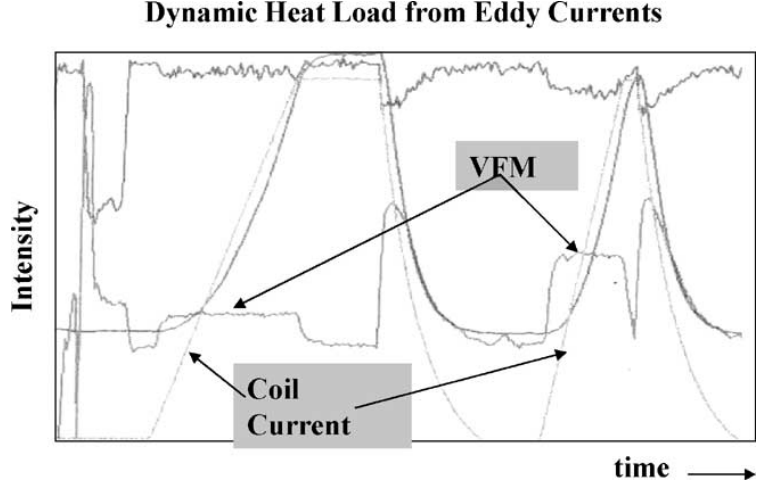

Fig. 8. In the graph the response signal from VFM can be seen, as observed when changing the current in the coil. The magnet was charged at constant current, i.e., at an induced constant power.

from the heat conduction through the various supports between the cold mass and the shield. It was also noticed that the average temperature difference, between the cold mass and the LHe, increased linearly with the shield temperature. Furthermore, the temperature gradient between the casing and the cooling pipes showed a similar linear increase, when changing the average shield temperature from $50 \mathrm{~K}$ to $88 \mathrm{~K}$.

\section{DyNAMic HeAT LOAD IN B0}

A superconducting magnet has, at $4.5 \mathrm{~K}$, an increased heat load, when changing the current in the coils, i.e., during the charge of the magnet (ramp up) and during the discharge (slow dump). In the case of B 0 and the BT coils, the additional losses are mostly due to eddy currents induced in the Al alloy coil casing, which acts as the secondary of a transformer, with one single turn in short circuit. Hysteresis and coupling losses in the superconductor have been estimated to be negligible as well as the eddy current losses in the pure $\mathrm{Al}$ cladding of the conductor. Accurate calculations of the power deposition in the coil casing have shown that the power has an uniform distribution, with only some marginal concentration in the area between the doublepancake, which enables a simple macroscopic model to be used.

The B0 magnet was charged at different rates $d I / d t$ from 10 to $18.8 \mathrm{~A} / \mathrm{s}$, each change at a constant power.

A few values where also collected when discharging the magnet into an external resistor, i.e., with the exponential current decay. The response signal from the VFM can be seen in Fig. 8. The high stability of the signal and its fast response time should be noticed.

The experimental data are shown in Fig. 9, where they are compared to the theoretical curves of the power P [W] in (2), which are calculated for two different equivalent resistance values of the coil casing.

$$
P[W]=\frac{\left[\frac{M}{n} * \frac{d I}{d t}\right]^{2}}{R_{c a \sin g}} .
$$

The longitudinal resistance $\mathrm{R}_{\text {casing }}$ for $\mathrm{B} 0$, assumed as one turn, is estimated to be 5.9-6.3 $\mu \Omega$. The casing mutual inductance with the coil $\mathrm{M}$ is assumed to be equal to the B0 coil inductance, i.e., $220 \mathrm{mH}$. $\mathrm{n}$ is ratio between the 120 turns for the two double-pancakes of B0, and the single turn for the casing. 
Heat Load due to Eddy Currents

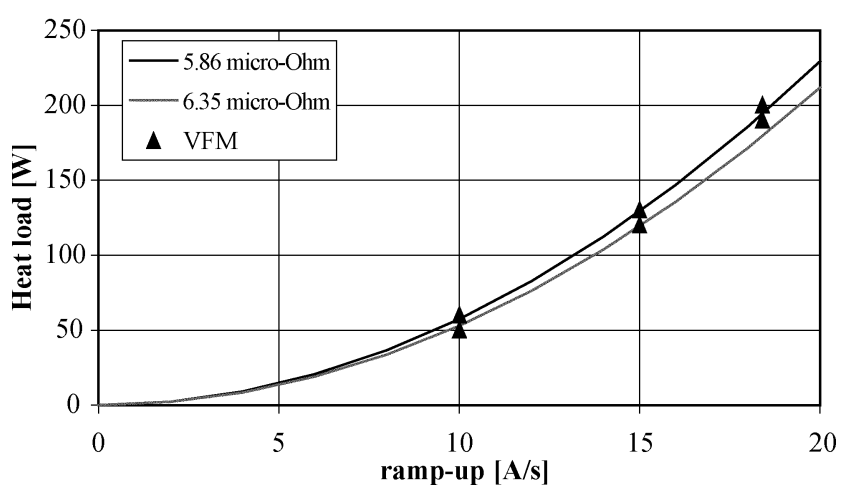

Fig. 9. Experimental values of the dynamic heat load versus current ramp rate. The two lines correspond to the theoretical values as per (2).

The results in Fig. 9 are very useful since they allow for the estimation of the heat load as seen by the refrigerator, during a ramp-up or a slow dump for the entire BT toroidal magnet, since the final slow dump will also be carried out at a constant power. The design value for the BT magnet system discharge is $4.2 \mathrm{~A} / \mathrm{s}$.

\section{CONCLUSION}

The use of a Void Fraction Meter (VFM) inserted on the return transfer line from the B0 magnet allowed carrying out pre- cise measurements on the heat load of its cold mass. Thanks to VFM fast time response and stable signal, it was also possible to measure the dynamic heat loads due to the eddy currents induced on the coil casing when changing the current in the coil, and to compare the results to the calculations.

\section{ACKNOWLEDGMENT}

The authors would like to thank M. A. Rodriguez Ruiz and $\mathrm{R}$. Losito for their valuable assistance in the signal analysis of the VFM.

\section{REFERENCES}

[1] D. Hagedorn, D. Leroy, P. Dullenkopf, and W. Haas, "Monitor for the quality factor in two-phase helium," Adv. Cryog. Eng., pp. 1288-1307, 1985.

[2] A. Daël et al., "Synthesis of technological developments for the B0 model coil and the ATLAS barrel toroid coils," IEEE Trans. Appl. Supercond., vol. 10, no. 1, pp. 361-364, March 2001.

[3] N. Delruelle et al., "First cryogenic testing of the ATLAS superconducting prototype magnets," IEEE Trans. Appl. Supercond., vol. 12, no. 1, pp. 1336-1338, March 2001.

[4] - "The common cryogenic test facility for the ATLAS barrel and end cap toroid magnet," in CEC/ICMC 2003, to be published.

[5] C. Mayri, private communication, CEA-SACLAY report 5C-2900T-\# 6000-007-PA, May 2003. 